\title{
Instability of the interface in two-layer flows with large viscosity contrast at small Reynolds numbers
}

\author{
Jiebin Liu' ${ }^{1,2}$ • Jifu Zhou ${ }^{1,2}$
}

Received: 11 January 2016 / Revised: 29 February 2016 / Accepted: 6 June 2016 / Published online: 30 September 2016 (C) The Chinese Society of Theoretical and Applied Mechanics; Institute of Mechanics, Chinese Academy of Sciences and Springer-Verlag Berlin Heidelberg 2016

\begin{abstract}
The Kelvin-Helmholtz instability is believed to be the dominant instability mechanism for free shear flows at large Reynolds numbers. At small Reynolds numbers, a new instability mode is identified when the temporal instability of parallel viscous two fluid mixing layers is extended to current-fluid mud systems by considering a composite error function velocity profile. The new mode is caused by the large viscosity difference between the two fluids. This interfacial mode exists when the fluid mud boundary layer is sufficiently thin. Its performance is different from that of the KelvinHelmholtz mode. This mode has not yet been reported for interface instability problems with large viscosity contrasts. These results are essential for further stability analysis of flows relevant to the breaking up of this type of interface.
\end{abstract}

Keywords Mud · Interface instability · Kelvin-Helmholtz instability $\cdot$ Interfacial modes

\section{Introduction}

Two-layer flows with a clear interface can be found in environmental and industrial flow problems. It is well known that the breaking of the interface is an important mechanism for mass and momentum transfer between the two layers. The present work has been motivated by the need to understand

\section{Jifu Zhou}

Zhoujf@imech.ac.cn

1 Key Laboratory for Mechanics in Fluid Solid Coupling Systems, Institute of Mechanics, Chinese Academy of Sciences, Beijing 100190, China

2 School of Engineering Sciences, University of Chinese Academy of Sciences, Beijing 100049, China the critical breaking condition of the interface between the fluid mud and the water. Although the parameters, such as density ratio and viscosity, are prescribed for fluid mud and water systems, the conclusion may be extended to other twolayer flows systems, if the controlling parameters drop in the similar range.

Fluid mud exists in estuaries and coastal areas. Its behaviors are of significant importance for waterway and harbor construction and protection. It is defined as a dense suspension of primarily cohesive particles of fine sediments and organic matter, with a sharp vertical density gradient at the mud-water interface $[1,2]$, which is for the first time termed "lutocline" by Parker and Kirby [3]. Its density ranges from $1050-1200 \mathrm{~kg} / \mathrm{m}^{3}$ and its dynamic viscosity varies from ten to one thousand times that of water [4], depending on its composition. Laboratory experiments have shown that fluid mud behaves like Bingham fluid. However, in real circumstances, it is always in motion due to the action of tides, currents and surface water waves. Hence, its Bingham property is not obvious, and it can be treated as a Newtonian fluid [5]. As a matter of fact, there are a lot of papers taking the fluid mud as Newtonian fluid in theoretical analysis or numerical simulation [6-11]. The cohesive fine sediments make fluid mud a structural integrity rather than a mixture of isolated particles. Consequently, the fluid mud is not suspended one particle by another, but by a group of particles like a plume flow as a result of the breaking of the lutocline, namely the mud-water interface. Therefore, it is very necessary to study the instability of the interface.

The motion of the interface plays an important role in the mass and momentum exchange in the vertical direction. Once the interface breaks, considerable fluid mud is brought into the water column. Current is usually considered to be one of 
the dynamic causes of the mud-water interface breaking and mass transport.

It has been found experimentally that several processes exist for interface breaking. At the initial stage when the current velocity is low, the interface is smooth and discernible. When the current velocity increases, interfacial waves appear gradually and propagate progressively downstream. If the current velocity increases further, the interfacial waves become shorter and steeper until they break at a critical current velocity when mixing begins. These processes are similar to those of water-sugar solutions studied experimentally by Keulegan [12].

The breaking of interfaces between two fluids is termed a hydrodynamic instability problem. This type of two-fluid parallel flow is termed a mixing layer. Its velocity profile, usually approximated by a hyperbolic tangent or error function as the base flow, is susceptible to Kelvin-Helmholtz $(\mathrm{K}-\mathrm{H})$ instability. The mixing layer stability was firstly studied theoretically by Helmholtz and later by Kelvin to explain the formation of ocean surface waves by wind. For a steady, twodimensional, parallel, uniform, two-fluid shear layer, flow becomes unstable when the velocity difference is sufficiently high, the density difference is sufficiently small, or the perturbation wave is sufficiently short [13]. In the $\mathrm{K}-\mathrm{H}$ model, a velocity jump exists across the interface, which is consistent with the case that the viscosity is not considered.

The $\mathrm{K}-\mathrm{H}$ model was then extended to include viscosity, which is present in real fluids $[14,15]$. Viscosity reduces the growth rate and cutoff wave number $\left(k_{c} \approx 1\right.$, where $k_{c}$ is the wave number beyond which no unstable mode exists, see Ref. [14]). However, the hyperbolic tangent base flow functions that were adopted are an approximate solution of the NavierStokes equations. Recently, the exact solution of the first Stokes problem was chosen as the base flow for instability analysis $[16,17]$. The dimensionless growth rate of the K$\mathrm{H}$ instability was nearly independent of Reynolds number [18]. The dimensional growth rate is therefore proportional to the velocity difference and inversely proportional to the vorticity thickness [17]. The $\mathrm{K}-\mathrm{H}$ mode has proven to be a generic instability of shear flow as the Reynolds number tends to infinity (page 116 in Ref. [18]).

As the Reynolds number approaches to infinity, the instability is assumed to develop more rapidly than the vortex layers. When the Reynolds number is small $(R e \approx 0)$, the growth rate decreases with Reynolds number, viscosity thickens the vorticity layer and competition occurs between instability growth and spreading because of viscosity. This leads to a correction in growth rate provided by inviscid analysis [19]. However, the analysis in Ref. [19] is incomplete because it only considered the dissipative effect of viscosity. Viscosity is believed to cause some instability modes in two-layer Couette and Poiseuille flows, such as the "interfacial mode" $[20,21]$ at small Reynolds numbers
$(R e \approx 0)$ and the "shear mode" [22,23] at sufficiently large Reynolds numbers, which depends on flow conditions.

The interest of most researchers has focused on the growth rate, rather than the critical Reynolds number, because flow becomes unstable at a relatively low Reynolds number. For example, for two-layer flow with uniform density, the critical Reynolds number is zero [13] (from which we can infer that stable density stratification has stabilizing effect on the $\mathrm{K}-\mathrm{H}$ flow, and the critical Reynolds number may be a small number approaching zero). When the critical condition for the onset of instability is investigated, small Reynolds numbers should be considered in instability analysis. For fluids such as fluid mud and water with large viscosity differences, it is natural to enquire how viscosity affects parallel viscous two-fluid mixing layer instability when the Reynolds number is small.

Exponential growth of infinitesimal perturbations to the basic parallel flow is the first of several processes that leads to the interface breaking. To obtain a complete physical understanding of the process, each step of the process must be understood. In this paper, linear stability analysis and nonlinear numerical simulation are used to investigate the stability of the mud-water interface at small Reynolds numbers. Linear theory is based on the assumption that the disturbances are infinitesimal, the interactions between instability waves are typically ignored, and the development of the mean flow is independent of the growth of the disturbances. These limitations exclude its application to the further development of disturbances, because the nonlinear effects become appreciable. In this stage, nonlinear numerical simulation should be deployed to account for nonlinear effects, including the generation of higher harmonics of the instability waves and the modification to the mean flow. The combination of linear stability analysis and nonlinear numerical simulation will provide valuable insight into early dynamics and a full description of the development of two-fluid shear flow, which help understand the instability mechanisms of the fluid mudwater interface.

In summary, by using linear instability analysis and nonlinear numerical simulation, we have studied the instability of the interface between fluid mud and water at small Reynolds numbers. We have demonstrated that the viscosity difference causes an important unstable short-wave interfacial mode, which has never been reported. This short-wave mode is different from the long-wave mode occurring at small Reynolds number [21] and the short-wave mode occurring at high Reynolds number [23]. We have further discussed the effects of viscosity and density ratio of the two fluids to the evolution of this short-wave mode.

This paper is organized as follows. Firstly, we formulate the problem and introduce the dimensionless number. Then, we describe the linear stability analysis (Sect. 3) and the techniques for the nonlinear numerical simulations (Sect. 4). In 


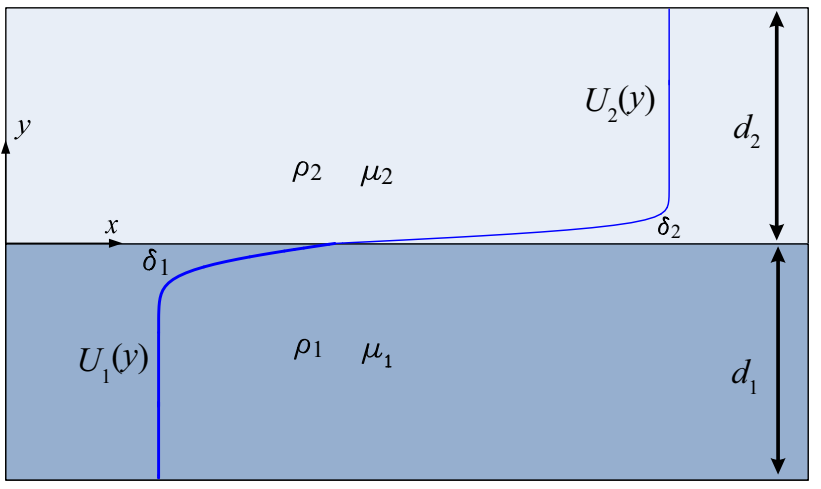

Fig. 1 Basic velocity profiles in the current and fluid mud

Sect. 5, we use these two methods to study the instability modes caused by viscosity contrast at small Reynolds numbers, especially the one that has an overwhelming growth rate. Finally, we summarize our results and present some conclusions.

\section{Problem formulation}

The flow configuration is shown schematically in Fig. 1. Coordinates $x$ and $y$ are located parallel and perpendicular to the undisturbed interface, respectively, with the origin of $y$ located at the interface. The lower layer of thickness $d_{1}$ is fluid mud with density $\rho_{1}$ and viscosity $\mu_{1}$. The upper layer of thickness $d_{2}$ is water with density $\rho_{2}$ and viscosity $\mu_{2}$. We consider the case where current is initiated rapidly, inducing two boundary layers above and beneath the interface, with the thickness given by $\delta_{j}=3.6 \sqrt{\nu_{j} t}(j=1,2)$, where $t$ is the time and $v_{j}$ is the kinematic viscosity in the upper and lower layers, respectively. This is termed the first Stokes problem, and the boundary thickness can be determined from the boundary layer equations [24]. The example for this problem is the experiment carried out by Thorpe [25], which is often used to illustrate the $\mathrm{K}-\mathrm{H}$ instability [26].

The exact solution of the first Stokes problem is given by [16]

$U_{1}(y)=U_{1}^{*} \operatorname{erf}\left(y / \delta_{1}\right), \quad y<0$,

$U_{2}(y)=U_{2}^{*} \operatorname{erf}\left(y / \delta_{2}\right), \quad y>0$,

where $\operatorname{erf}(x)=\frac{2}{\sqrt{\pi}} \int_{0}^{x} \mathrm{e}^{-\xi^{2}} \mathrm{~d} \xi$ and $U_{j}(j=1,2)$ represent the asymptotic velocities, which are related by the continuity of tangential stress on the interface, i.e.,

$\mu_{2} \frac{\partial U_{2}}{\partial y}=\mu_{1} \frac{\partial U_{1}}{\partial y}, \quad$ at $\quad y=0$.

We take the velocity amplitude in the fluid mud layer $U_{1}^{*}$ (hereinafter denoted as $U_{0}$ ) to measure the velocity, and the boundary thickness in fluid mud $\delta_{1}$ as the length scale, and introduce the following dimensionless parameters

$$
\begin{aligned}
& r=\frac{\rho_{1}}{\rho_{2}}, m=\frac{\mu_{1}}{\mu_{2}}, n_{1}=\frac{d_{1}}{\delta_{1}}, n_{2}=\frac{d_{2}}{\delta_{2}}, \\
& \operatorname{Re}=\frac{\rho_{1} U_{0} \delta_{1}}{\mu_{1}}, F r=\frac{\rho_{1} U_{0}^{2}}{\left(\rho_{1}-\rho_{2}\right) g \delta_{1}}, S=\frac{\rho_{1} U_{0}^{2} \delta_{1}}{T},
\end{aligned}
$$

where $r$ and $m$ represent density and viscosity ratios, respectively, $R e$ is the Reynolds number of fluid mud flow, $F r$ is the Froude number, $S$ is the Weber number, and $T$ is the interfacial tension.

From Eq. (3) we can infer that:

$\frac{U_{2}^{*}}{U_{1}^{*}}=\frac{\mu_{1}}{\mu_{2}} \frac{\delta_{2}}{\delta_{1}}=m \frac{3.6 \sqrt{\nu_{2} t}}{3.6 \sqrt{\nu_{1} t}}=m \frac{\sqrt{\mu_{2} / \rho_{2}}}{\sqrt{\mu_{1} / \rho_{1}}}=m \sqrt{\frac{r}{m}}=\sqrt{m r}$.

Similarly, the current Reynolds number defined by $R e_{2}=$ $\frac{\rho_{2} U_{2}^{*} \delta_{2}}{\mu_{2}}$ is related to the fluid mud Reynolds number by:

$R e_{2}=\frac{m U_{2}^{*} \delta_{2}}{r U_{1}^{*} \delta_{1}} R e=\frac{m \sqrt{m r} \sqrt{r / m}}{r} R e=m R e$.

\section{Linear temporal stability analysis}

The exact solution of the first Stokes problem is chosen as the base flow of the mixing layer, which is shown in Eqs. (1) and (2).

Introducing the following scales, $(u, v)=U_{0}\left(u^{\prime}, v^{\prime}\right)$, $(x, y)=\delta_{1}\left(x^{\prime}, y^{\prime}\right), p=\rho_{1} U_{0}^{2} p^{\prime}, t=\left(\delta_{1} / U_{0}\right) t^{\prime}$, we nondimensionalize the system. Here, the variables with prime denote the dimensionless variables. In the following, the primes are dropped. The stability of the parallel two flow layers is investigated by disturbing the base flow infinitesimally [27]. We assume that the flow is incompressible, and introduce the stream functions $\psi_{j}$ to represent the disturbance velocities $\left(\hat{u}_{j}, \hat{v}_{j}\right)$ in the fluids so that $\left(\hat{u}_{j}, \hat{v}_{j}\right)=$ $\left(\partial \psi_{j} / \partial y,-\partial \psi_{j} / \partial x\right)$.

We write:

$\psi_{j}(x, y, t)=\phi_{j}(y) \mathrm{e}^{\mathrm{i} k(x-c t)}$,

where $\mathrm{i}$ is the imaginary unit, $k$ is a real wave number, $c$ is a complex wave speed, and $\phi$ which is known as the eigenfunction represents the amplitude of the disturbance. The real part of $c$ determines the phase velocity, and the imaginary part, $c_{i}$, provides the growth rate $\omega_{i}=c_{i} k$.

Substitution of the stream functions into the linearized Navier-Stokes equations results in the Orr-Sommerfeld equations in dimensionless form:

$$
\begin{aligned}
& \left(\mathrm{D}^{2}-k^{2}\right)^{2} \phi_{1}+\mathrm{i} k \operatorname{Re}\left[U_{1}\left(k^{2}-\mathrm{D}^{2}\right)+\mathrm{D}^{2} U_{1}\right] \phi_{1} \\
& \quad=\mathrm{i} c k \operatorname{Re}\left(k^{2}-\mathrm{D}^{2}\right) \phi_{1},
\end{aligned}
$$


for the fluid mud, $-n_{1}<y<0$, and

$$
\begin{aligned}
& \left(\mathrm{D}^{2}-k^{2}\right)^{2} \phi_{2}+\mathrm{i} k \operatorname{Re} \frac{m}{r}\left[U_{2}\left(k^{2}-\mathrm{D}^{2}\right)+\mathrm{D}^{2} U_{2}\right] \phi_{2} \\
& =\mathrm{i} c k \operatorname{Re} \frac{m}{r}\left(k^{2}-\mathrm{D}^{2}\right) \phi_{2},
\end{aligned}
$$

for the water, $0<y<n_{2}$, where $\mathrm{D}$ is the derivative operator $\mathrm{d} / \mathrm{d} y$.

The linearized interface conditions imposed at the interface $y=0$ provide five expressions as follows [21,28]

$$
\begin{aligned}
& \hat{\eta}\left(c-U_{1}\right)-\phi_{1}=0, \\
& \phi_{1}=\phi_{2}, \\
& \mathrm{D} \phi_{1}+\mathrm{D} U_{1} \hat{\eta}=\mathrm{D} \phi_{2}+\mathrm{D} U_{2} \hat{\eta}, \\
& \mathrm{D}^{2} \phi_{1}+k^{2} \phi_{1}+\mathrm{D}^{2} U_{1} \hat{\eta}=\left(\mathrm{D}^{2} \phi_{2}+k^{2} \phi_{2}+\mathrm{D}^{2} U_{2} \hat{\eta}\right) / m, \\
& \left(\mathrm{D}^{3}-3 k^{2} \mathrm{D}\right) \phi_{1}+\mathrm{i} k \operatorname{Re}\left[\left(c-U_{1}\right) \mathrm{D}+\mathrm{D} U_{1}\right] \phi_{1} \\
& \quad=\left(\mathrm{D}^{3}-3 k^{2} \mathrm{D}\right) \phi_{2} / m+\mathrm{i} k \operatorname{Re}\left[\left(c-U_{2}\right) \mathrm{D}+\mathrm{D} U_{2}\right] \phi_{2} / r \\
& \quad+\mathrm{i} k \operatorname{Re}\left(F r^{-1}+k^{2} S^{-1}\right) \hat{\eta},
\end{aligned}
$$

where $\hat{\eta}$ is the amplitude of the interfacial displacement given by $\eta=\hat{\eta} \mathrm{e}^{\mathrm{i}(k x-\omega t)}$.

The boundary conditions at the lower wall are nopenetration and no-slip, which read:

$\phi_{1}=\mathrm{D} \phi_{1}=0, y=-n_{1}$,

and the disturbances can be ignored far from the interface, which leads to:

$\phi_{2}=\mathrm{D} \phi_{2}=0, y=n_{2}$.

The stability problem described by the governing differential equations together with the conditions at the boundaries and the interface represents a generalized eigenvalue problem, in which the wave speed $c$ is the eigenvalue.

A Chebyshev collocation algorithm [29] is used to evaluate the eigenvalue problem. Once the $c$ is given, the growth rate $\omega_{i}=c_{i} k$ can be obtained too. If $\omega_{i}>0$, the flow is unstable, otherwise it is stable or neutrally stable. Our code was validated by comparing our result with that of Yecko et al. (from Fig. 2a in Ref. [16]), as shown in Fig. 2 demonstrating an excellent agreement.

In Sect. 5, we will do linear stability analysis of mudwater interface by means of this code itself. We will further do nonlinear analysis by means of nonlinear numerical simulation (as described in the following section) for the evolution of the most unstable mode identified by this linear analysis code.

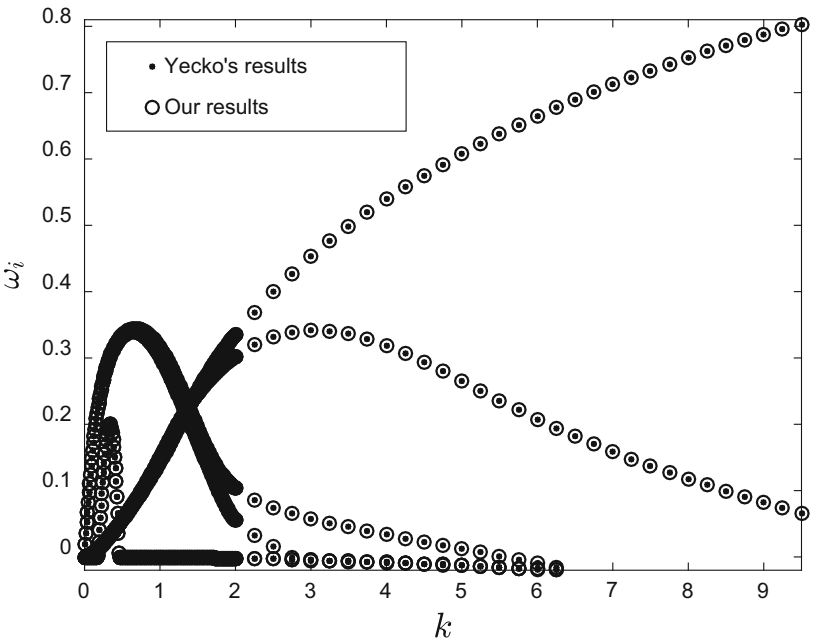

Fig. 2 Comparison of results between Yecko [16] and our model: $R e=$ 2000, $\mathrm{Fr}^{-1}=0, m=50, r=500, \delta_{1} / \delta_{2}=1$

\section{Nonlinear numerical simulations}

One of the objectives of this work is to study the nonlinear evolution of unstable modes using a numerical model. In order to directly compare the evolution patterns of the disturbance wave obtained by our code with those in the previous literature, the results from the nonlinear numerical simulation are shown in dimensional form. However, it will be converted to dimensionless form if compared with the results of linear stability analysis. Correspondingly, the governing equations are dimensional.

\subsection{Governing equations}

The flows are governed by free-divergence and variabledensity (viscosity) incompressible Navier-Stokes equations, and the convective equation for cell volume fraction:

$\nabla \cdot \boldsymbol{u}^{*}=0$

$\frac{\partial \boldsymbol{u}^{*}}{\partial t^{*}}+\left(\boldsymbol{u}^{*} \cdot \nabla\right) \boldsymbol{u}^{*}=-\frac{\nabla p}{\rho}+\boldsymbol{g}+\frac{\mu}{\rho} \Delta \boldsymbol{u}^{*}$,

$\frac{\partial F}{\partial t^{*}}+\boldsymbol{u}^{*} \cdot \nabla F=0$

where $\boldsymbol{u}^{*}=\left(u^{*}, v^{*}\right)$ is the dimensional velocity vector, $t^{*}$ is the dimensional time, $\boldsymbol{g}$ is the gravitational acceleration, $F$ is the volume ratio occupied by fluid mud in a cell, $\rho$ and $\mu$ are the density and the dynamic viscosity of each cell, respectively, which are determined by the following expressions:

$\rho=\rho_{1} F+\rho_{2}(1-F)$,

$\mu=\mu_{1} F+\mu_{2}(1-F)$. 


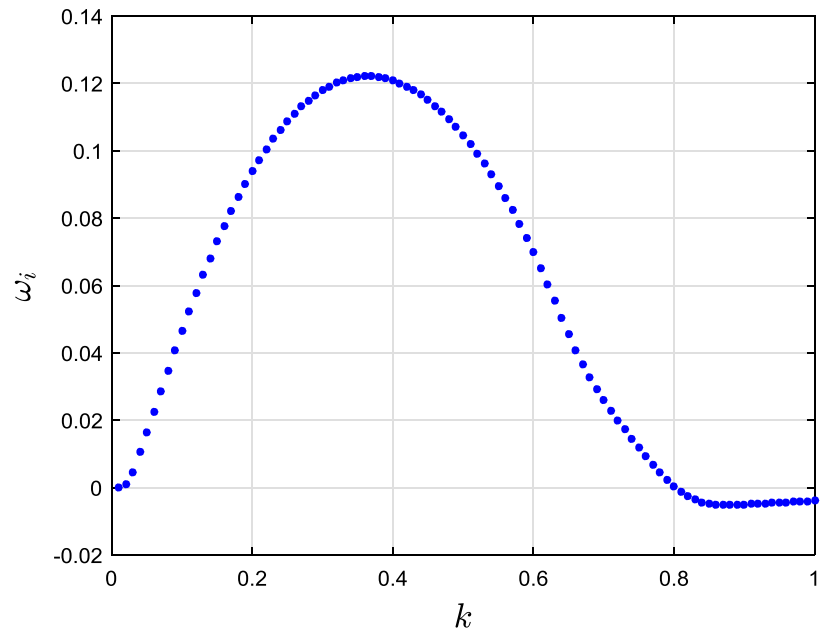

Fig. 3 The growth rate of $\mathrm{K}-\mathrm{H}$ instability versus wave number at large Reynolds numbers predicted by linear stability analysis. $\rho_{2}=$ $1000 \mathrm{~kg} / \mathrm{m}^{3}, \mu_{2}=9.7 \times 10^{-4} \mathrm{~Pa} \cdot \mathrm{s}, \delta_{1}=0.01 \mathrm{~m}, U_{2}^{*}=1.09 \mathrm{~m} / \mathrm{s}, r=$ $1.02, m=1, n_{1}=10, n_{2}=12$

It should be noted that the variables in the governing equations are dimensional.

\subsection{Numerical procedure}

To solve these equations numerically, the velocity and pressure variables are allocated on a staggered marker-and-cell Eulerian grid. The delaying modified QUICK scheme is used to deal with convective terms of momentum equations. The time discretization is the fully-implicit Euler backward difference. The viscosity terms are approached by central difference. The standard "pressure implicit with splitting of operator scheme" of Issa et al. [30] was used to solve the Navier-Stokes equations. The divergence-free phenomenon is satisfied by one prediction and two correction steps at each time step. The velocity is then used as the initial velocity condition at the following time step. The volume of fluid function is updated when the correct velocity field is gained. Because of the sharp jump in $F$ across the interface, a geometry construction algorithm proposed by Rudman [31] and Youngs [32] is implemented to reconstruct the interface and update the volume fraction function.

The eigenfunctions from the linear stability analysis are used to initialize the interface and velocity profile [33]. Given that temporal instability is considered, a spatially periodic condition is implemented in the stream direction [34], and the interface is tracked by the volume of fluid function.

\subsection{Code validation}

To validate our code, we simulated the evolution of $\mathrm{K}-\mathrm{H}$ instability at a large Reynolds number $(R e \approx 11349)$. The density and viscosity of the upper layer are $\rho_{2}=1000 \mathrm{~kg} / \mathrm{m}^{3}$ and $\mu_{2}=9.7 \times 10^{-4} \mathrm{~Pa} \cdot \mathrm{s}$, respectively. The density and viscosity ratio are chosen as $r=1.02$ and $m=1$. The initial boundary thickness and free stream velocity are chosen as $\delta_{1}=0.01 \mathrm{~m}$ and $U_{2}^{*}=1.09 \mathrm{~m} / \mathrm{s}$, and the dimensionless thicknesses of the upper and lower layers are fixed as $n_{1}=10$ and $n_{2}=12$. These parameters were chosen to ensure that the flow regime approximates a classical $\mathrm{K}-\mathrm{H}$ instability. The result from linear stability analysis shows that the most dangerous mode is the $\mathrm{K}-\mathrm{H}$ mode, which has a growth rate of $\omega_{i}=0.1221$ corresponding to a wave number $k=0.37$, as shown in Fig. 3 . The disturbance velocities derived from the stream function of the most dangerous mode is used as the initial field of the nonlinear numerical simulation. The simulation was performed with $400 \times 150$ grids, and four waves were considered in the computational box. The periodic boundary condition is enforced in the stream direction for the temporal analysis.

Figure 4 shows that the disturbance wave grows with time, suggesting that the base flow is unstable. As expected, the evolution of the $\mathrm{K}-\mathrm{H}$ mode is very close to that of the classical $\mathrm{K}-\mathrm{H}$ instability (page $21-22$ in Ref. [26], the last page in Ref. [25], page 6 in Ref. [35]). Initially, strong shear exists between two fluids separated by the interface. When a perturbation is added to the interface, the subsequent action is for the asymmetric velocity distribution to increase the size of the disturbances, which increases the instability further and generates overturning rolls.

Given that temporal instability is considered, $\eta=$ $\hat{\eta} \mathrm{e}^{\mathrm{i} k(x-c t)}=\hat{\eta} \mathrm{e}^{\omega_{i} t} \mathrm{e}^{\mathrm{i} k\left(x-c_{r} t\right)}$, the disturbance will propagate with an envelope $\hat{\eta} \mathrm{e}^{\omega_{i} t}$, which grows with time following $\mathrm{e}^{\omega_{i} t}$. Let us assume that an observer standing at $x=0$ sees the crest of the waves at one moment. After a time $2 \pi /\left(c_{r} k\right)$ (where $c_{r}$ is the phase speed of the disturbance waves), he/she will see a crest with amplitude of $\hat{\eta} \mathrm{e}^{\omega_{i} t}$ again. Figure 5 gives a time series of the crest elevation. It can be seen that the linear theory therefore predicts very well the initial exponential growth of small perturbations. However, as the wave begins to roll (e.g., at $t=10,25$, or so), the observer will measure two elevations of the interface at the same time. In Fig. 5, only the higher elevation is plotted, so the dotted line is discontinuous. When the interface breaks, linear theory does not apply and nonlinear numerical simulation provides a good picture of the nonlinear evolution.

\section{Results and discussion}

Bearing the properties of fluid mud in mind, we chose the density and viscosity of water as $\rho_{2}=1000 \mathrm{~kg} / \mathrm{m}^{3}$ and $\mu_{2}=$ $9.7 \times 10^{-4} \mathrm{~Pa} \cdot \mathrm{s}$ and use the following parameter value ranges: $r=1-1.2, m=6.25-16000, \operatorname{Re}=0.01-3$, and $F r^{-1}=$ 10-60, which spans a broad range of water and fluid mud 

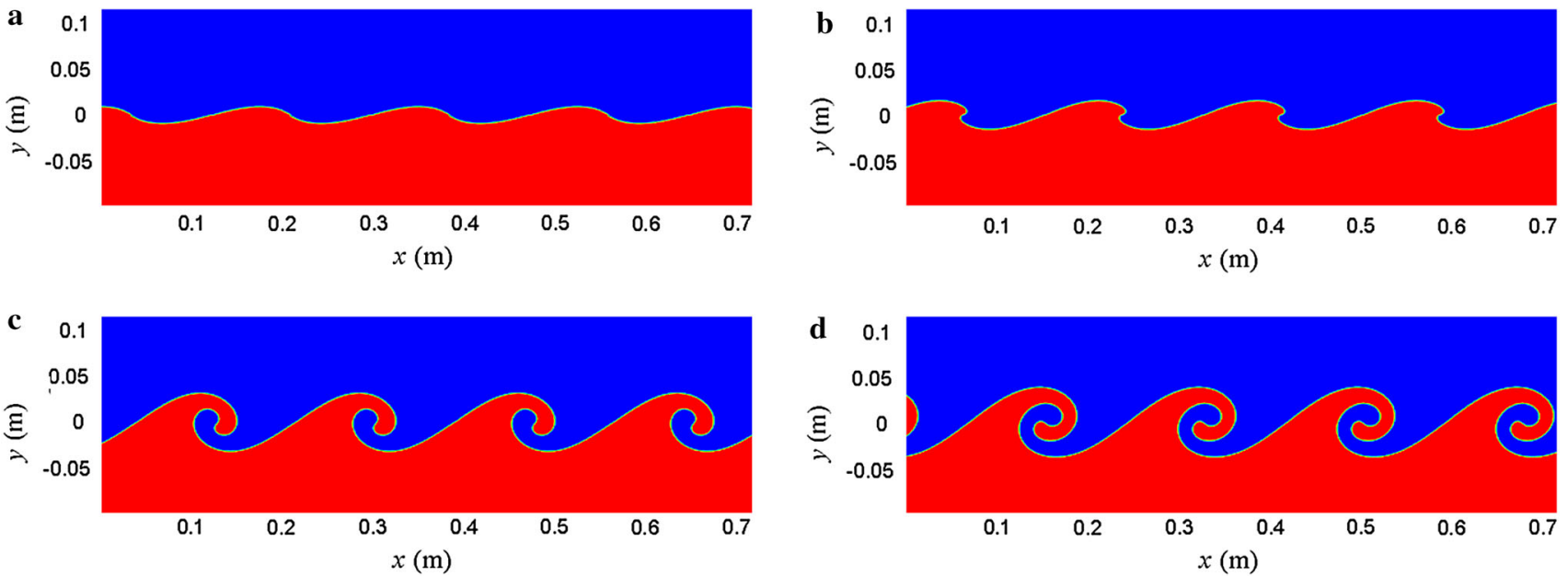

Fig. 4 Simulation of K-H instability resulting in overturning billows at the interface between two fluids. $\rho_{2}=1000 \mathrm{~kg} / \mathrm{m}^{3}, \mu_{2}=9.7 \times 10^{-4} \mathrm{~Pa} \cdot \mathrm{s}$, $\delta_{1}=0.01 \mathrm{~m}, U_{2}^{*}=1.09 \mathrm{~m} / \mathrm{s}, r=1.02, m=1, n_{1}=10, n_{2}=12 . \mathbf{a} t^{*}=0.1139 \mathrm{~s} . \mathbf{b} t^{*}=0.1423 \mathrm{~s} . \mathbf{c} t^{*}=0.1913 \mathrm{~s} . \mathbf{d} t^{*}=0.2277 \mathrm{~s}$

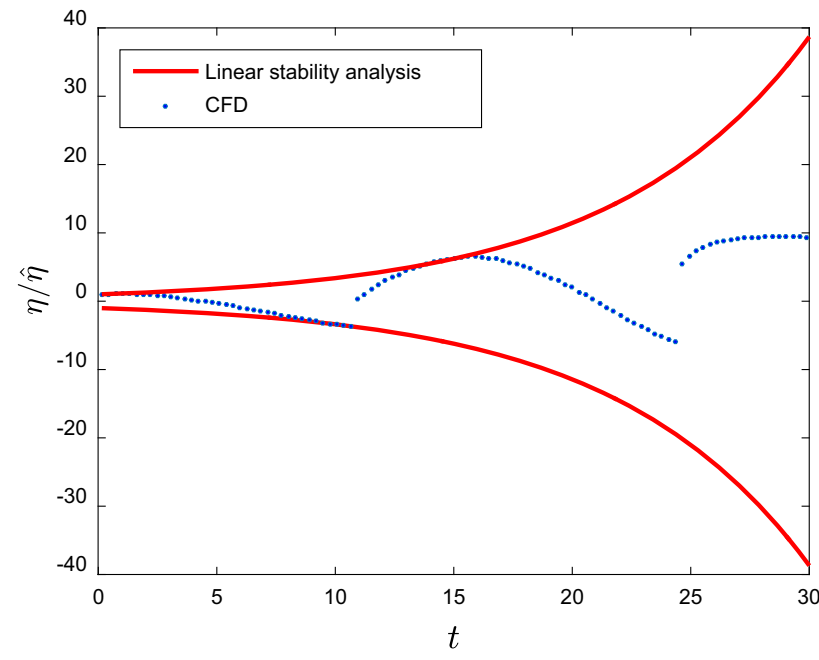

Fig. 5 Time evolution of the interface position at $x=0$ (dotted curve), i.e., $\hat{\eta} \mathrm{e}^{\omega_{i} t} \mathrm{e}^{-\mathrm{i} k c_{r} t}$ and envelope predicted by linear theory (solid curve), i.e., $\hat{\eta} \mathrm{e}^{\omega_{i} t}$, where the growth rate is given by linear theory

combinations. If the density and viscosity of the fluid mud are specified as $\rho_{1}=1200 \mathrm{~kg} / \mathrm{m}^{3}$ and $\mu_{1}=1 \mathrm{~Pa} \cdot \mathrm{s}$, the above ranges of $R e$ and $F r$ correspond to $U_{2}^{*}=0.25-2.6 \mathrm{~m} / \mathrm{s}$ and $\delta_{1}=0.002-0.083 \mathrm{~m}$. It should be noted that the results in all the following figures, except for figures of disturbance wave evolution, are given by linear stability analysis.

The calculations show that the fluid mud layer thickness and water layer depth have little influence on stability if $n_{1} \geq$ $3, n_{2} \geq 8$. When $n_{1}, n_{2} \leq 3$, the growth rate of the long wave mode resulted from the viscosity mismatch is sensitive to the thickness (see Fig. 6).

Calculations show (see Fig. 7) that surface tension acts as a stabilizing factor for all modes, as shown previously by Hooper and Boyd [20] and Yecko et al. [16]. In Fig. 7, we can see that the flow is always unstable, but the increase of surface tension decreases the growth rate and does not change the dominance of the unstable modes. To study the most unstable case and for simplicity as well, the following analysis excludes surface tension effects.

\subsection{Unstable interfacial modes}

By choosing particular flow parameters, Yecko et al. [16] demonstrated the presence of mode competition at a large Reynolds number $(R e=2000)$, where the dominant unstable mode can vary between different modes. Our linear analysis shows that mode competition also occurs at low Reynolds number, as shown in Fig. 8a, in which different modes dominate over different ranges of wave number. Four modes exist in Fig. 8, which are identified in the present paper as "long wave", "K-H mode", "mode III", and "mode IV". They are distinguished between each other by different wave numbers at which the growth rate arrives at its extreme, implying the most unstable wave number in its local vicinity. The former two modes can be seen more clearly in Fig. 8b.

In Fig. 9, we present the vertical profiles of the eigenfunctions for the four most dangerous wave numbers (i.e., $k=0.015,0.275,1.1$, and 2.5) corresponding to the four most unstable modes shown in Fig. 8a. It can be seen that the maximum absolute values of the real and imaginary parts of the eigenfunctions of all modes occur at the interface $(y=0)$, suggesting that all modes are interfacial modes.

Except for $\mathrm{K}-\mathrm{H}$ mode, other modes are caused by viscosity difference, because the growth rate of disturbance will decay to zero (see Fig. 3 ) as the wave number exceeds the cut off wave number $\left(k_{c} \approx 1\right)$ due to viscosity dissipation for two flow layers without viscosity difference [14,15], and stable density stratification usually suppresses the instability. 

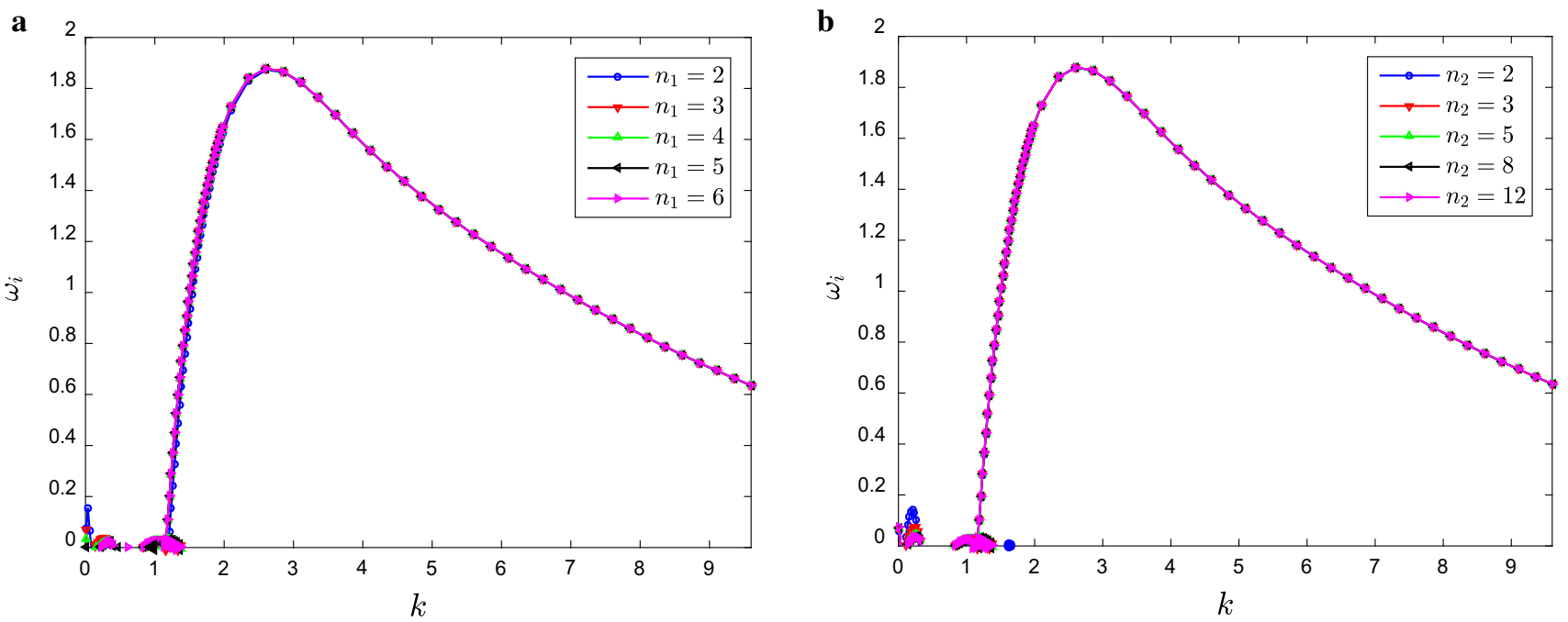

Fig. 6 The effect of calculated thickness of the two layers on the growth rate. $R e=0.75, F r^{-1}=95, S=0, m=1000, r=1.2$. a $n_{2}=8$. b $n_{1}=3$

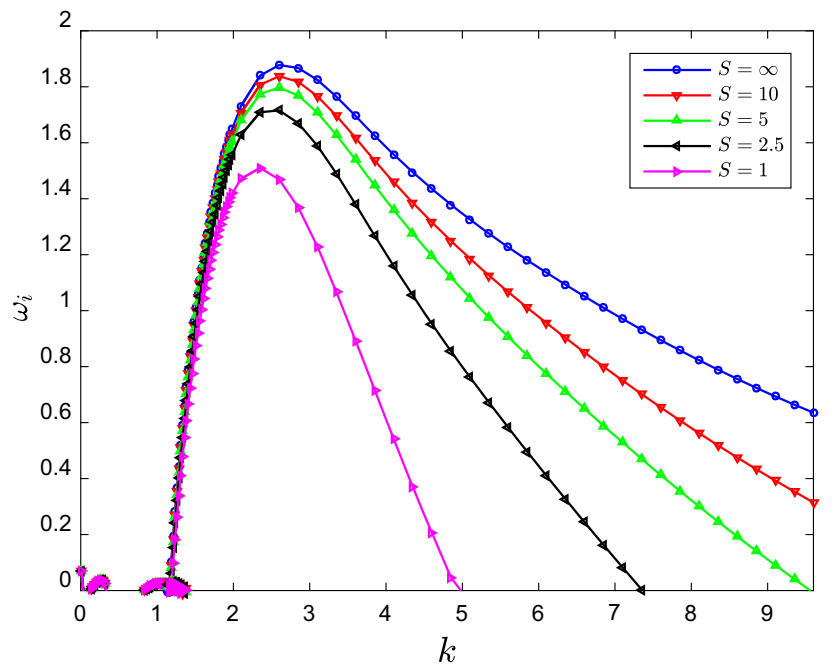

Fig. 7 The effect of surface tension on the growth rate. $R e=0.75$, $F r^{-1}=95, m=1000, r=1.2, n_{1}=3$, and $n_{2}=8$

\subsection{Effect of viscosity difference}

Viscosity stratification may yield interfacial mode instabilities as first identified by Yih [21] as a source of this instability in bounded two-layer plane Poiseuille and Couette flows. Yih showed that at arbitrarily small Reynolds numbers, the interface could become unstable to long waves because of viscosity stratification. Later, a short-wavelength interfacial mode was identified in the subsequent papers, such as bounded Couette flow [20] and unbounded plane Poiseuille flow [22].

To illustrate the effect of viscosity difference on the interfacial modes of mud-water flows, which are different from plane Poiseuille flow, we calculate the growth rates ver- sus wave number for different viscosity contrast at different Reynolds numbers and Froude numbers, as shown in Figs. 10 and 11.

The difference between these two figures is of the scale and the parameters. In Fig. 10, the dimensional growth rate is scaled by $U_{0}, \delta_{1}$, and in Fig. 11, the growth rate is scaled by $U_{2}^{*}, \delta_{2}$. The dimensional growth rate $\omega_{i}^{*}$ can be expressed as $\omega_{i}^{*}=\omega_{i} U_{0} / \delta_{1}=\omega_{i 2} U_{2}^{*} / \delta_{2}$, where $\omega_{i 2}$ denotes the growth rate in dimensionless form by scaling length with $\delta_{2}$, velocity with $U_{2}^{*}$. So, $\omega_{i 2}$ relates to $\omega_{i}$ by $\omega_{i 2}=\omega_{i} / m$. It will be convenient to compare the growth rate with the results in Refs. [14,15], where the growth rate was scaled in this way.

In Fig. 10, the density ratio is set to 1.2 and the mud Reynolds number is fixed at 0.75 . As the viscosity ratio decreases, the growth rates of all modes decrease. It can be expected that the growth rate will become negative at a certain viscosity ratio. In fact, the growth rate decays to zero if $m<700$ for the parameters given in this figure.

In Fig. 11, the density ratio is set to one and the water Reynolds number is fixed at 1000 . We can see that at the same current condition, mode IV appears for all viscosity ratios considered. This indicates that viscosity stratification alone is sufficient to cause instability.

In Figs. 8, 10, and 11, we can see that the growth rate of mode IV is the greatest. Hence, we focus on mode IV in the following discussions.

\subsection{Effect of density stratification}

Similarly, the effect of density ratio on the growth rate of different modes can be gained. It can be seen from Fig. 12 that the variation in density has only a small influence on the growth rate of mode IV. The mode IV is always unstable 

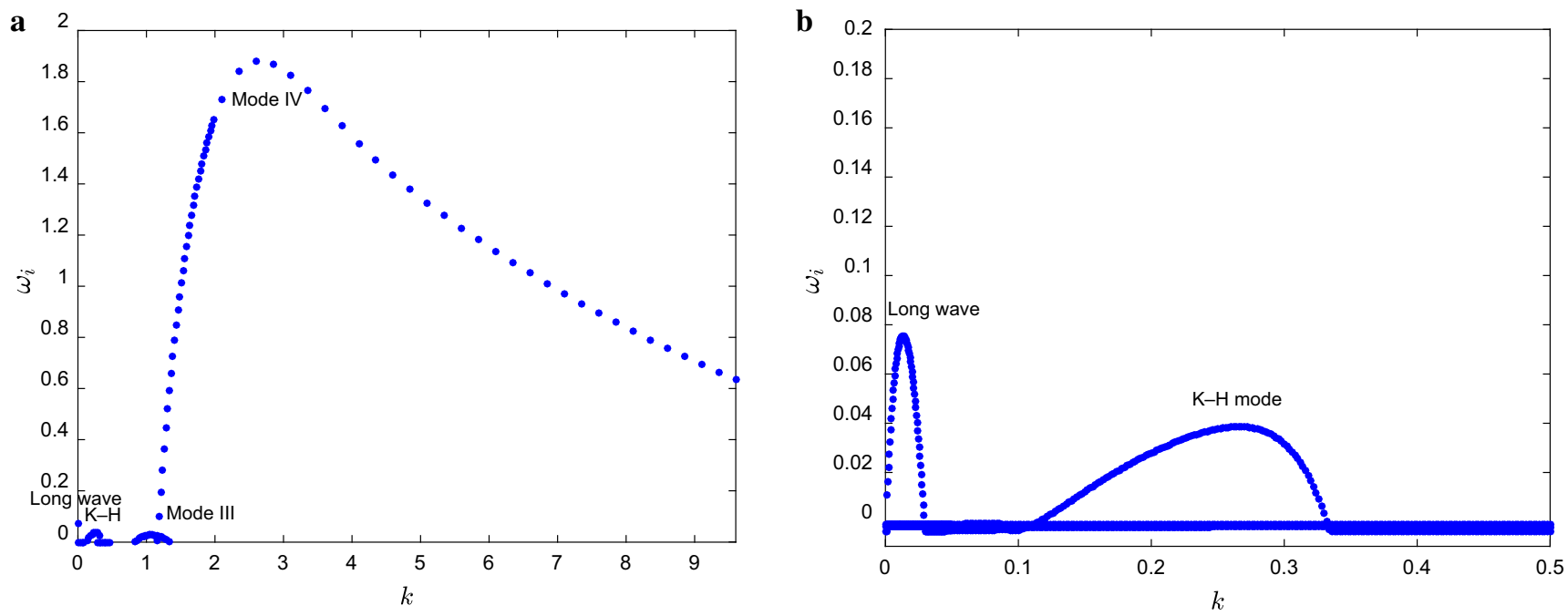

Fig. 8 Growth rates of different modes as a function of wave number. a Mode competition. $\mathbf{b}$ An enlargement of a at small wave number. $R e=0.75$, $F r^{-1}=95, m=1000, r=1.2, n_{1}=3$, and $n_{2}=8$
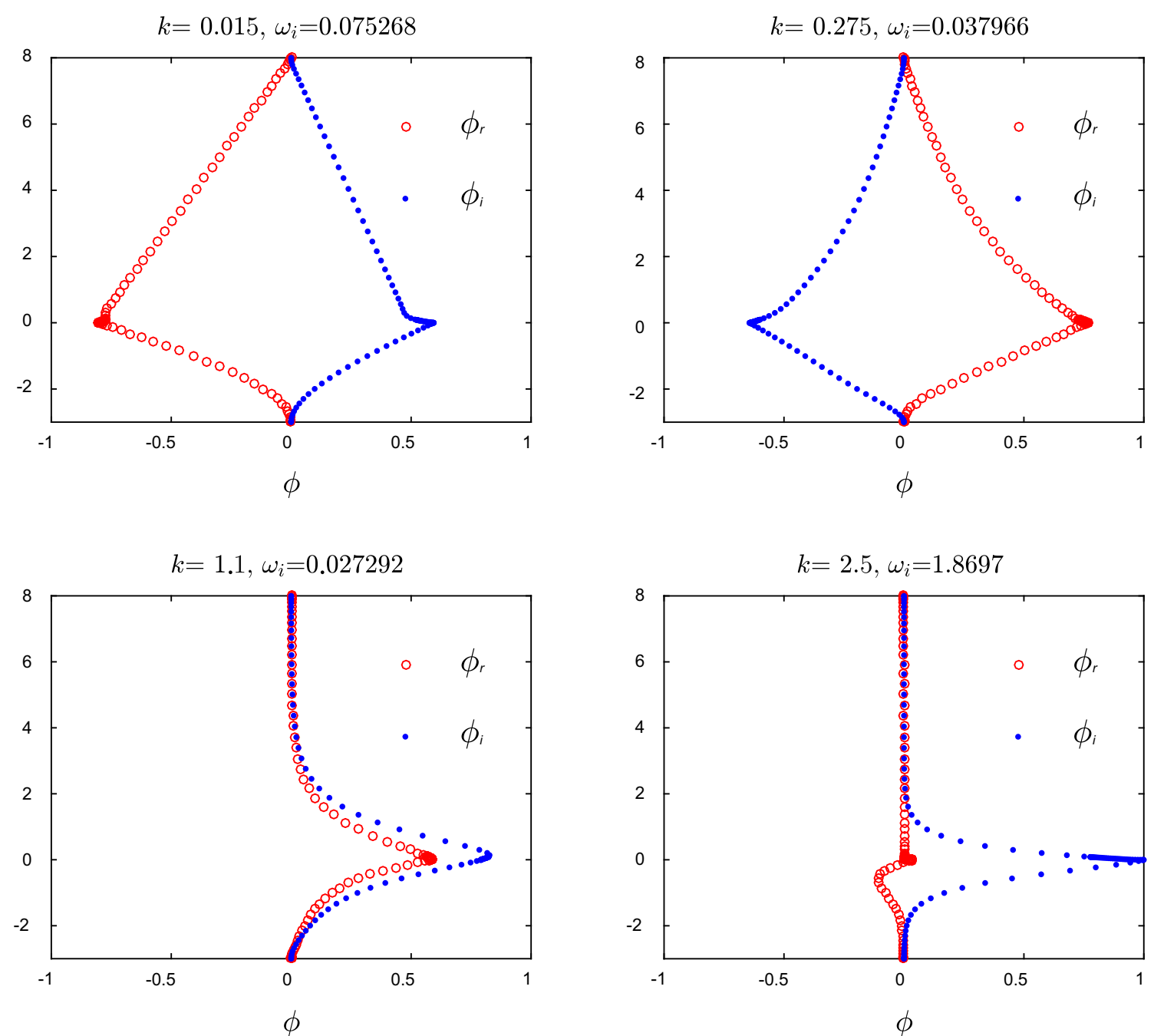

Fig. 9 Cross-stream structure of real and imaginary parts of $\phi$ associated with the most dangerous wave number for different modes in Fig. $8 \mathrm{a}$ 


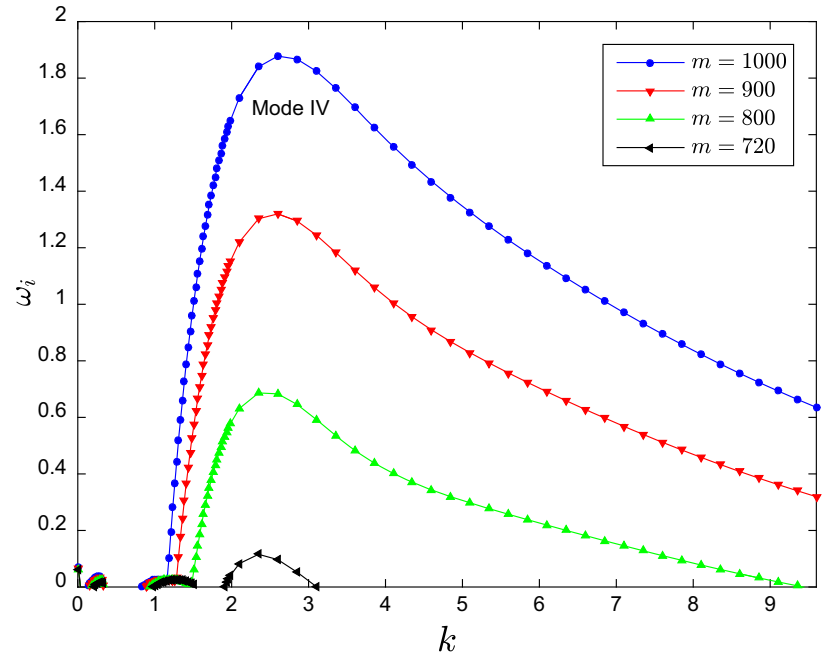

Fig. 10 The effect of viscosity difference on the growth rate versus wave number: $R e=0.75, F r^{-1}=95, r=1.2, n_{1}=3$, and $n_{2}=8$

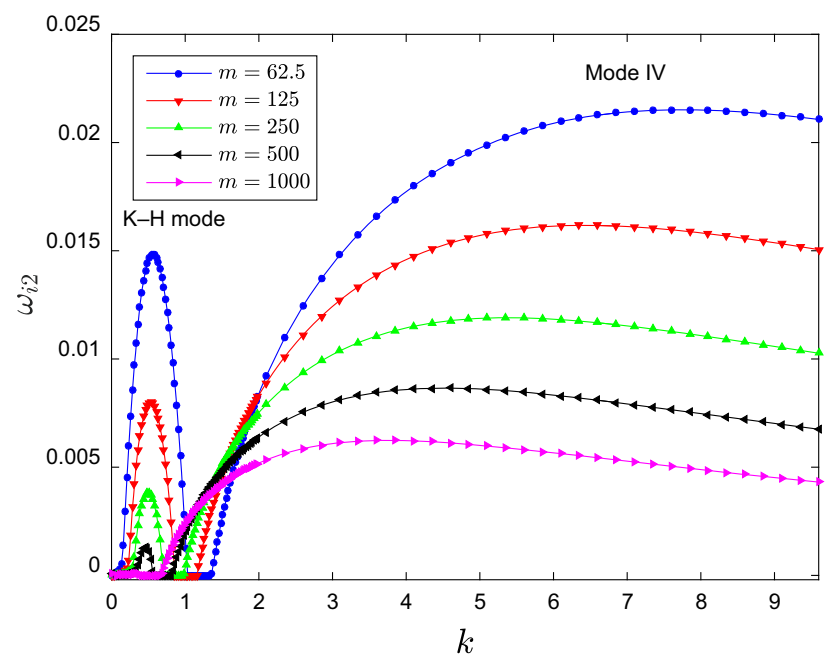

Fig. 11 The effect of viscosity difference on the growth rate versus wave number for equal density fluids: $R e_{2}=1000, \mathrm{Fr}^{-1}=0, r=$ $1, n_{1}=3$, and $n_{2}=8$

for all density ratios considered here. It is rational to infer that, once the viscosity ratio is large enough, for example, $m=1000$, no matter how the density ratio changes, the mode IV is always unstable. In order words, the density ratio does not govern the instability, at least for mode IV.

However, the destabilizing effect of viscosity stratification may be overshadowed by the stabilizing effect of density stratification, which has also been noted by Yih [21]. In fact, mode IV can emerge only if the viscosity contrast is sufficiently strong, which can be inferred from Fig. 13. For example, mode IV will become stable if $m \leq 500$. However, the growth rate does not vary monotonously with $m$, as shown in Fig. 13.

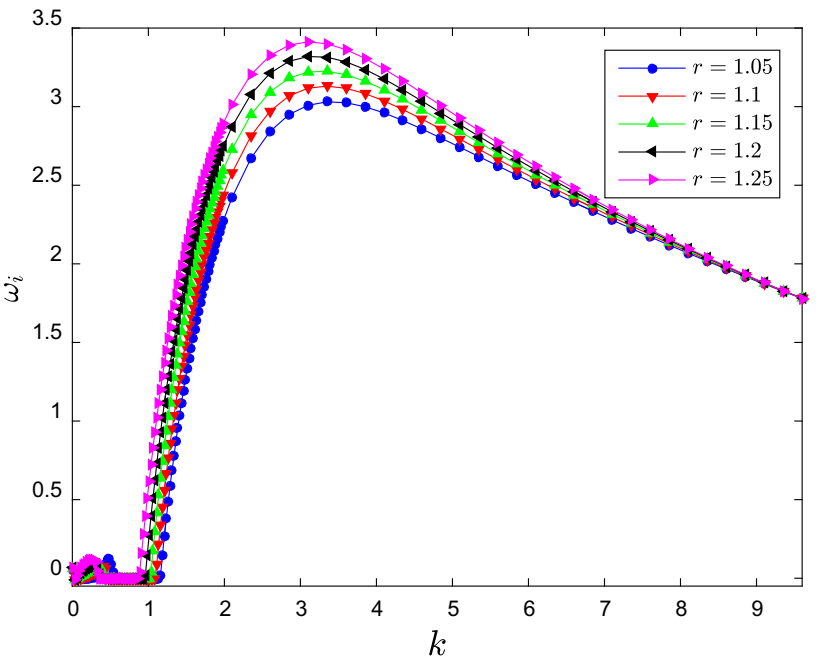

Fig. 12 Effect of density ratio on growth rate of mode IV: $R e=$ $1, F r^{-1}=60, m=1000, n_{1}=3$, and $n_{2}=8$

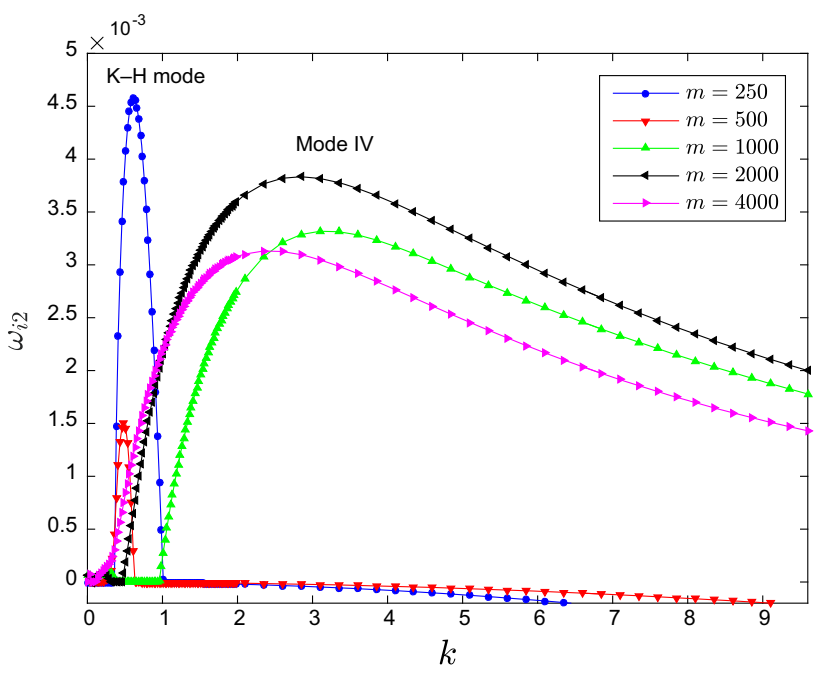

Fig. 13 Growth rate of different modes as a function of wave number for different values of $m: R e_{2}=1000, F r^{-1}=60, r=1.2, n_{1}=3$, and $n_{2}=8$

\subsection{Effect of Froude number and Reynolds number}

The dimensionless parameter that measures the stabilizing effect of density stratification is the Froude number $(F r)$. In Fig. 14, we provide the growth rate of mode IV as a function of $\mathrm{Fr}$ for different $\mathrm{Re}$. The growth rate decreases as $\mathrm{Fr}$ decreases, indicating that the increase of fluid mud boundary thickness results in a more stable flow regime. This is consistent with the previous knowledge.

The effect of Reynolds number on different modes is shown in Fig. 15. As the Reynolds number is increased, the $\mathrm{K}-\mathrm{H}$ mode arises while mode IV disappears. This excludes the inertia as the candidate mechanism to cause the unstable 


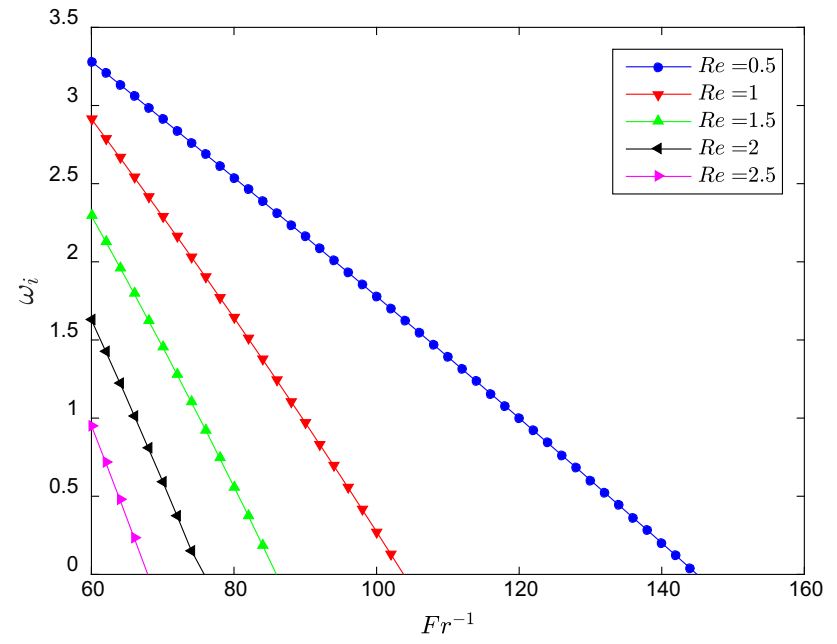

Fig. 14 Growth rates of mode IV at the most unstable wave number as a function of Froude and Reynolds numbers: $m=1000, r=1.2$, $n_{1}=3$, and $n_{2}=8$

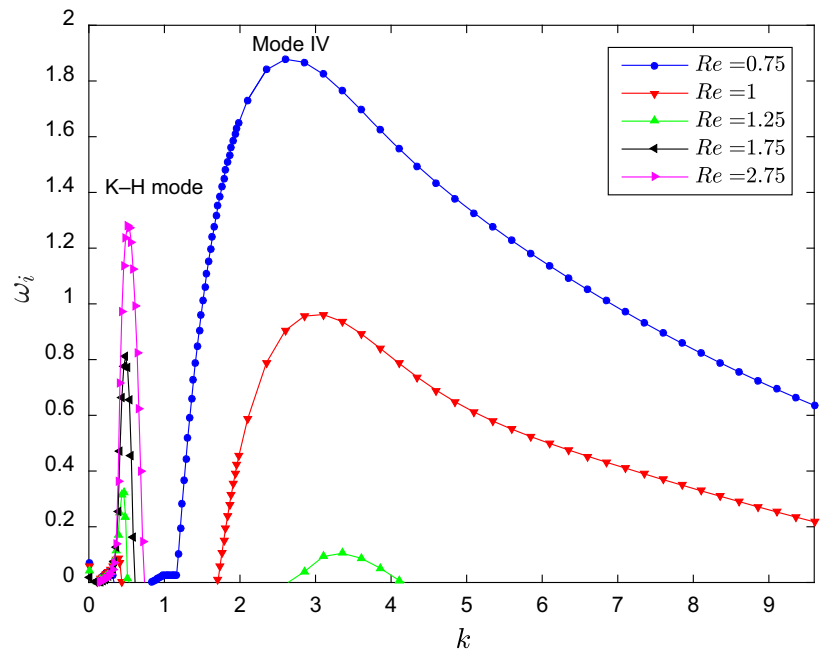

Fig. 15 The effect of $\operatorname{Re}$ on different modes for $r=1.2, m=1000$, $F r^{-1}=95, n_{1}=3$, and $n_{2}=8$.

mode IV. So, it is once again that mode IV is caused by the viscosity difference.

\subsection{Nonlinear evolution initialized by the most unstable mode}

To investigate the role of mode IV in the dynamic process of the interface breaking, linear analysis offers little help. Hence, we next carry out nonlinear numerical simulations to account for its nonlinear evolutions.

Grid independence tests have been carried out to determine the adequate numerical resolution for the problem at hand. Three grid resolutions of $180 \times 360$ (coarse), $200 \times 400$ (medium), and $220 \times 440$ (fine) were tested. Very similar interfaces were found for the medium and fine grids (Fig. 16).
The grids of resolution $220 \times 440$, which is adopted in following simulation, is found fine enough to the simulation of all of the length scales of interest. To resolve the small scale structures, finer vertical grids with half spatial steps are used in the neighbor interval of the interface. The time step in time advancing is selected such that it is small enough to keep the numerical stability and large enough to reduce the numerical dissipation.

The simulation is seeded with the most unstable eigenmode. Two wavelengths are covered in the computational domain. Because the base flow is unstable, the disturbance waves will grow with time. Figure 17 shows six snapshots of the mud-water interface during its evolution until breaking. Since temporal instability is considered, only one of the waves will be discussed below. During the linear growth stage, the amplitude of the wave is small, and its crest and trough are asymmetric. As the amplitude increases, nonlinear distortion commences. The wave crest is stretched and a ligament is formed at the wave crest, which is then further stretched and carried downstream by the current. When the ligament is stretched to such a point that its width is sufficiently thin, it begins to break. This process differs from that of the $\mathrm{K}-\mathrm{H}$ instability, where a row of perturbations grows and rolls up into billows [26], and the ligament stretch is absent, as show in Fig. 4.

This nonlinear numerical simulation demonstrates that the interface breaking mechanism is a modified shear instability, which is strongly dependent on the viscosity contrast between mud and water, and is different from the $\mathrm{K}-\mathrm{H}$ mode, in which viscosity is found to reduce the growth rate and cutoff wavenumber [14].

\subsection{Neutral stability curves}

To investigate critical parameters of the interface instability, we calculate and plot the growth rate on the $\left(\delta_{1}, U_{2}^{*}\right)$ plane, as shown in Fig. 18. The $\mathrm{K}-\mathrm{H}$ mode dominates if the fluid mud boundary is thicker, whereas mode IV is more important if the fluid mud boundary is thinner. The $\mathrm{K}-\mathrm{H}$ mode has a lower growth rate, which distinguishes it from mode IV.

An overview of the stability properties for mode IV and the $\mathrm{K}-\mathrm{H}$ mode is also visible from the neutral stability curves on the (Re,k) plane, as shown in Fig. 19 for different Froude numbers. For each mode, the $(R e, k)$ plane is separated by the corresponding neutral curve into stable and unstable regions, which are denoted by $S$ and $U$, respectively. The colored $U$ identifies the unstable region limited by the neutral curves of the same color. As $F r$ decreases, the unstable region for mode IV on the $(R e, k)$ plane becomes narrow. This is consistent with the finding from Fig. 14. As $F r$ decreases, the critical Reynolds number for the $\mathrm{K}-\mathrm{H}$ mode increases. Stable density stratification therefore has a stabilizing effect on the $\mathrm{K}-\mathrm{H}$ mode. 

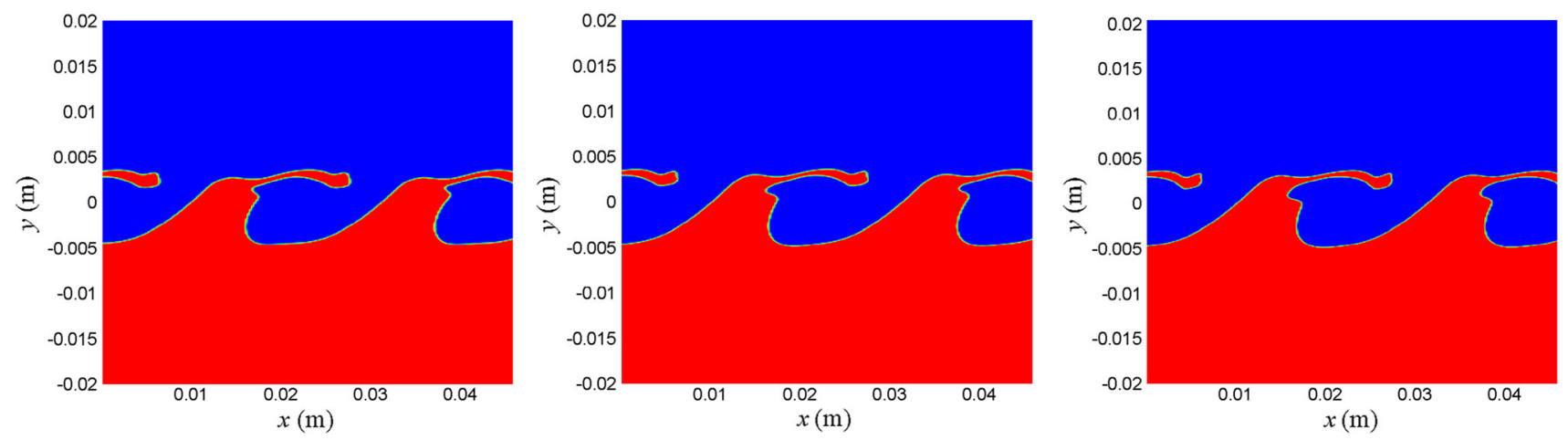

Fig. 16 Comparison of wave profile for different grid resolutions $180 \times 360$ (left), $200 \times 400$ (middle), and $220 \times 440($ right $)\left(U_{2}^{*}=2.09 \mathrm{~m} / \mathrm{s}, \delta_{1}=\right.$ $0.01 \mathrm{~m}, m=1000, r=1.2, n_{1}=4, n_{2}=5$, and $k=2.96$ )
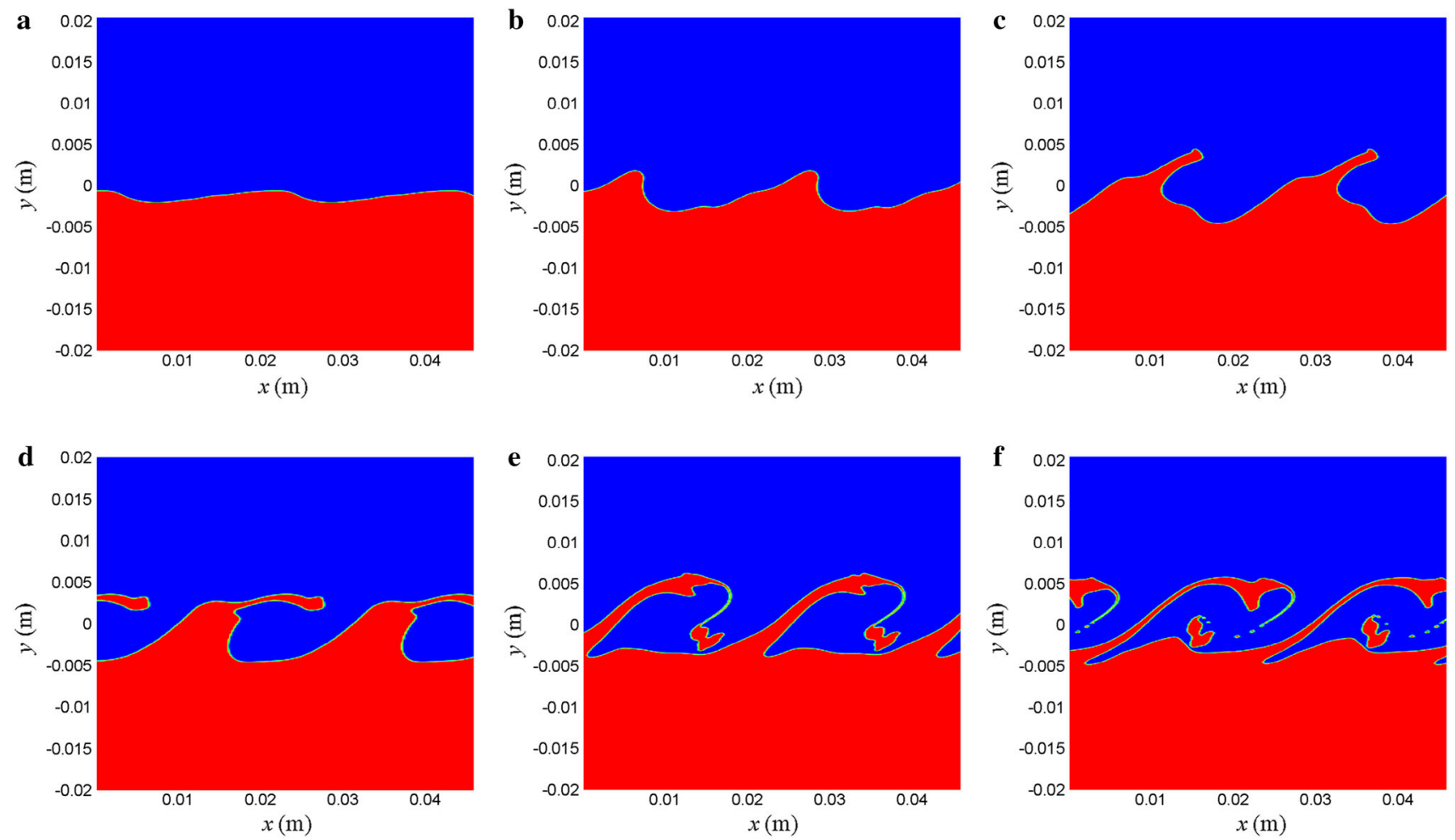

Fig. 17 Evolutions of mode IV: $U_{2}^{*}=2.09 \mathrm{~m} / \mathrm{s}, \mathrm{d}_{1}=0.01 \mathrm{~m}, R e=0.72, F r^{-1}=4.48, m=1000, r=1.2, n_{1}=1.2, n_{2}=5$, and $k=2.96$. $\mathbf{a} t^{*}=0.0117 \mathrm{~s} . \mathbf{b} t^{*}=0.0175 \mathrm{~s} . \mathbf{c} t^{*}=0.0234 \mathrm{~s}$. d $t^{*}=0.0292 \mathrm{~s} . \mathbf{e} t^{*}=0.0351 \mathrm{~s} . \mathbf{f} t^{*}=0.0468 \mathrm{~s}$

\section{Conclusions}

Motivated by explaining fluid mud resuspension mechanism under current forcing, we carried out linear stability analysis and nonlinear numerical simulations to account for nonlinear instability and explored the breaking mechanisms of the mud-water interface.

It is found that the viscosity contrast between mud and water causes multiple new interfacial modes at small Reynolds numbers. One is the dominant instability mode, which is identified by mode IV. Its growth rate is signifi- cantly greater than the others, including the classical $\mathrm{K}-\mathrm{H}$ mode.

The behavior of this new mode is different from the classical $\mathrm{K}-\mathrm{H}$ mode. It depends strongly on the viscosity difference between mud and water. The growth rate of the new mode decreases linearly with Froude number. When the fluid mud boundary layer is thin and the free stream velocity is small, the new mode dominates the interface instability. Otherwise, the $\mathrm{K}-\mathrm{H}$ mode dominates. This is another type of mechanism of interface breakup under the forcing of currents, except for the $\mathrm{K}-\mathrm{H}$ mode. 

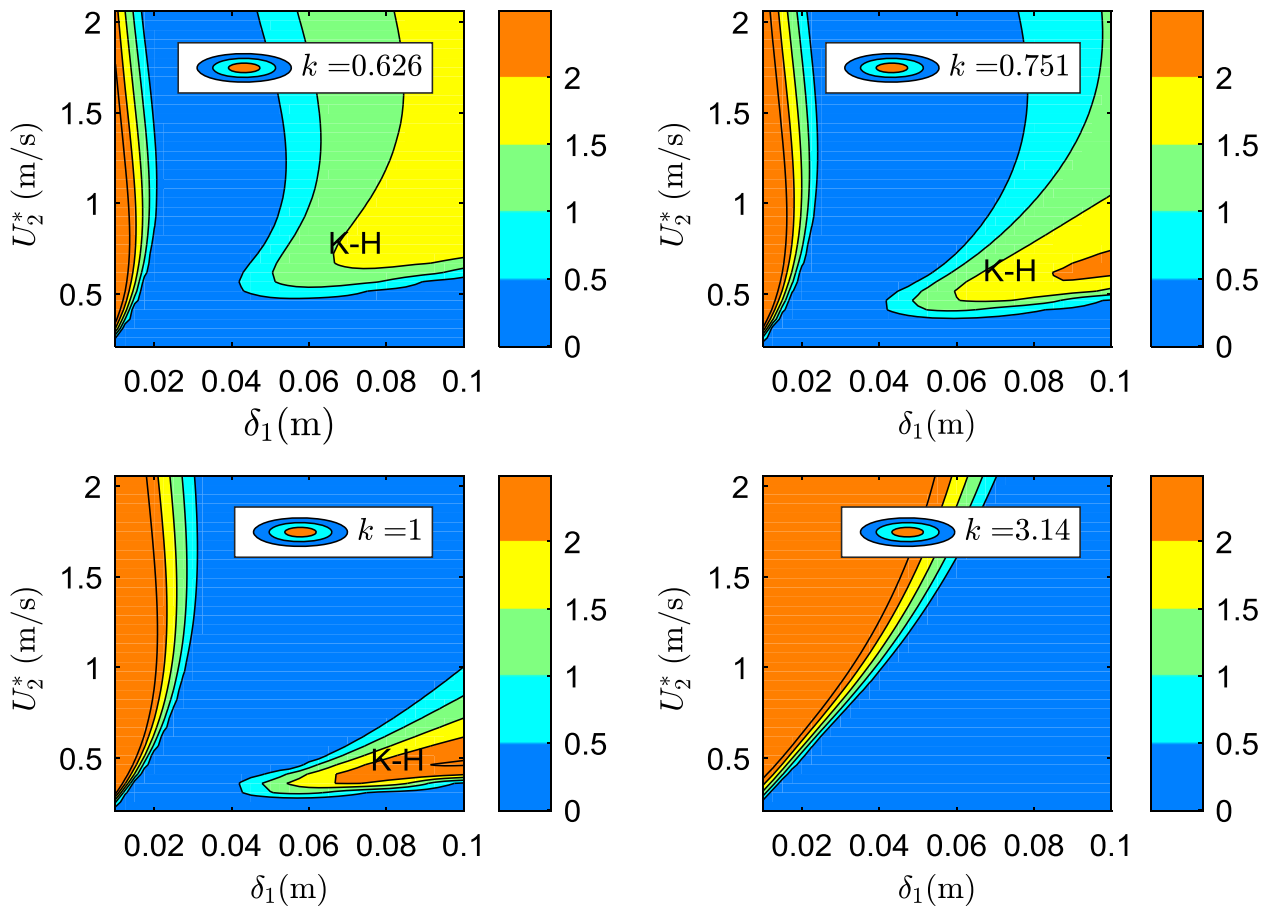

Fig. 18 (Color online) Contours of growth rate: $m=1000, r=1.2, n_{1}=3$, and $n_{2}=8$
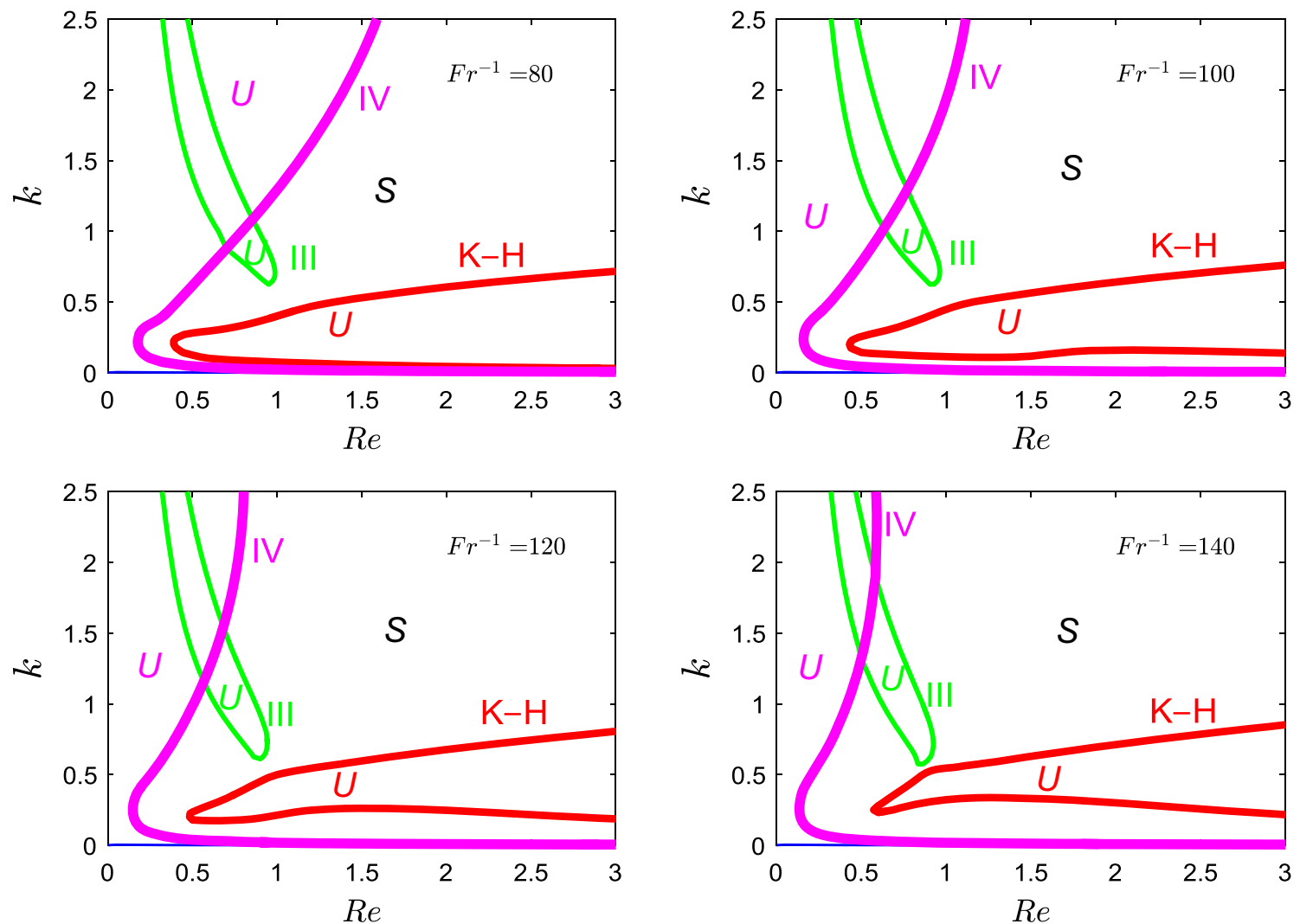

Fig. 19 Neutral stability diagram of different modes for different Froude numbers with stable and unstable regions denoted by $S$ and $U$, respectively: $m=1000, r=1.2, n_{1}=3$, and $n_{2}=8$ 
Acknowledgments The project was supported by the National Natural Science Foundation of China (Grants 11172307, 11232012, 11572332), 973 Program (Grant 2014CB046200), and the Strategic Priority Research Program of the Chinese Academy of Sciences (Grant XDB22040203).

\section{References}

1. Mcanally, W.H., Friedrichs, C., Hamilton, D., et al.: Management of fluid mud in estuaries, bays, and lakes. I: present state of understanding on character and behavior. J. Hydraul. Eng. 133, 9-22 (2007)

2. Mehta, A.J.: Understanding fluid mud in a dynamic environment. Geo Mar. Lett. 11, 113-118 (1991)

3. Parker, W.R., Kirby, R.: Time dependent properties of cohesive sediment relevant to sedimentation management-european experience. In: Estuarine Comparisons, Oregon, 573-589 (1982)

4. Zhao, Z.: Transformation of waves over fluid mud beds. Mar. Sci. Bull. 12(1), 1-9 (1993) (in Chinese)

5. Mehta, A.J.: On estuarine cohesive sediment suspension behavior. J. Geophys. Res. 94, 14303-14314 (1989)

6. Gade, H.G.: Effects of a nonrigid, impermeable bottom on plane surface waves in shallow water. J. Mar. Res. 16, 61-82 (1958)

7. Dalrymple, R.A., Liu, P.L.F.: Waves over soft muds: a two-layer fluid model. J. Phys. Oceanogr. 8, 1121-1131 (1978)

8. Sakakiyama, T., Bijker, E.W.: Mass-transport velocity in mud layer due to progressive waves. J Waterw. Port Coast. Ocean Eng. 115, 614-633 (1989)

9. Liu, P., Chan, I.C.: On long-wave propagation over a fluid-mud seabed. J. Fluid Mech. 579, 467-480 (2007)

10. Chiu-On, N.: Water waves over a muddy bed: a two-layer stokes' boundary layer model. Coast. Eng. 40, 221-242 (2000)

11. Hu, Y., Guo, X., Lu, X., et al.: Idealized numerical simulation of breaking water wave propagating over a viscous mud layer. Phys. Fluids 24, 802-805 (2012)

12. Keulegan, G.H.: Interfacial instability and mixing in stratified flows. J. Res. Natl. Bureau Stand. 43, 487-500 (1949)

13. Kundu, P.K., Cohen, I.M., Dowling, D.: Fluid Mechanics. Academic Press, New York (2012)

14. Betchov, R.: Stability of a shear layer between parallel streams. Phys. Fluids 6, 1391-1396 (1963)

15. Michalke, A.: On the inviscid instability of the hyperbolictangent velocity profile. J. Fluid Mech. 19, 543-556 (1964)

16. Yecko, P., Zaleski, S.P., Fullana, J.: Viscous modes in two-phase mixing layers. Phys. Fluids 14, 4115-4122 (2002)

17. Liu, J., Zhou, J.: The instability of water-mud interface in viscous two-layer flow with large viscosity contrast. Theor. Appl. Mech. Lett. 4, 062007 (2014)
18. Charru, F.O.: Hydrodynamic Instabilities. Cambridge University Press, Cambridge (2011)

19. Villermaux, E.: On the role of viscosity in shear instabilities. Phys. Fluids 10, 368-373 (1998)

20. Hooper, A.P., Boyd, W.: Shear-flow instability at the interface between two viscous fluids. J. Fluid Mech. 128, 507-528 (1983)

21. Yih, C.: Instability due to viscosity stratification. J. Fluid Mech. 27, 337-352 (1967)

22. Yiantsios, S.G., Higgins, B.G.: Linear stability of plane poiseuille flow of two superposed fluids. Phys. Fluids 31, 3225-3238 (1988)

23. Hooper, A.P., Boyd, W.G.C.: Shear-flow instability due to a wall and a viscosity discontinuity at the interface. J. Fluid Mech. 179, 201-225 (1987)

24. Schlichting, H., Gersten, K.: Boundary-Layer Theory. Springer, Berlin (2000)

25. Thorpe, S.A.: A method of producing a shear flow in stratified fluid. J. Fluid Mech. 32, 693-704 (1968)

26. Drazin, P.G., Reid, W.H.: Hydrodynamic Stability. Cambridge University Press, Cambridge (2004)

27. Chandrasekhar, S.: Hydrodynamic and Hydromagnetic Stability. Courier Dover Publications, Mineola (2013)

28. Boomkamp, P.: Classification of instabilities in parallel two-phase flow. Int. J. Multiph. Flow 22, 67-88 (1996)

29. Trefethen, L.N.: Spectral Methods in Matlab. Society for Industrial \& Applied Mathematics, Philadelphia (2000)

30. Issa, R.I., Ahmadi-Befrui, B., Beshay, K.R., et al.: Solution of the implicitly discretised reacting flow equations by operator-splitting. J. Comput. Phys. 93, 388-410 (1991)

31. Rudman, M.: Volume-tracking methods for interfacial flow calculations. Int. J. Numer. Methods Fluids 24, 671-691 (1997)

32. Youngs, D.L.: Time-dependent multi-material flow with large fluid distortion. Numer. Methods Fluid Dyn. 24, 273-285 (1982)

33. Valluri, P., Náraigh, L.Ó., Ding, H., et al.: Linear and nonlinear spatio-temporal instability in laminar two-layer flows. J. Fluid Mech. 656, 458-480 (2010)

34. Boeck, T., Li, J., López-Pagés, E., et al.: Ligament formation in sheared liquid-gas layers. Theoret. Comput. Fluid Dyn. 21, 59-76 (2007)

35. Fringer, O.B.: Numerical simulations of breaking interfacial waves. [Ph.D. Thesis], Stanford University, America (2003) 